\title{
A Stochastic System with Possible Maintenance of Standby Unit and Replacement of the Failed Unit Subject to Inspection
}

\author{
Anju Dhall \\ Department of Mathematics \\ G.G.D.S.D. College \\ Palwal, Haryana, India
}

\author{
S.C. Malik \\ Department of Statistics \\ M.D. University \\ Rohtak Haryana, India
}

\author{
V.J. Munday \\ Department of Statistics \\ M.D. University \\ Rohtak Haryana, India
}

\begin{abstract}
In this paper, a stochastic system of two identical units has been investigated by conducting possible maintenance of the cold standby unit before getting it into operation. A single repair facility is provided immediately to rectify the faults which occur during system operation. The inspection of the failed unit is done to see the feasibility of its repair. If repair of the unit is not feasible to the system, it is replaced immediately by new one. The random variables are statistically independent. The time to failure of the unit follows negative exponential distribution while the distributions for maintenance, inspection and repair times are taken as arbitrary with different probability density functions. The maintenance and repair of the unit are perfect. The semiMarkov process and regeneration point technique are used to derive the expressions for some reliability measures of vital significance. The graphical behavior of MTSF, Availability and profit function have been observed for particular values of different parameters and costs.
\end{abstract}

Keywords: Stochastic System, Possible Maintenance, Immediate Replacement, Inspection, Repair and Reliability Measures.

\section{INTRODUCTION}

No doubt that the technique of redundancy is one way to improve the performance of repairable systems but on the other hand it can be achieved by adopting proper maintenance policies. Therefore, stochastic models of standby systems have been studied extensively by the researchers including Gupta and Mumtaz (1996), Yadavalli et al. (2004), Bao and Cui (2012) and Kumar et al. (2012) under different sets of assumptions on failure and repair mechanisms. In most of these studies, it is commonly assumed that the cold standby unit can be brought into operation immediately at the failure of operating unit. But, this assumption seems to be unrealistic when a system has to operate in varying environmental conditions. The standby unit may deteriorate because of its non functionality for a long period of time. Thus, in such a situation, possible maintenance of the standby unit may be done before getting it into operation. Also, repair of the failed unit may or may not be feasible to the system and this fact can be revealed by inspection. Recently, Malik and Barak (2013), Malik (2013) and Yusuf and Yusuf (2014) evaluated performance measures of cold standby systems with maintenance and repair.

The aim of the present study is to analyze a stochastic system of two identical units in which one unit is initially operative and the other is kept as spare in cold standby. There is a single server who attends the system immediately as and when needed. The standby unit undergoes for possible maintenance with some probabilities when it is not found good at the failure of operating unit. The inspection of the failed unit is done to see the feasibility of its repair. If repair of the unit is not feasible to the system, it is replaced immediately by new one. The random variables are statistically independent. The time to failure of the unit follows negative exponential distribution while the distributions for maintenance, inspection and repair times are taken as arbitrary with different probability density functions. The maintenance and repair of the unit are perfect. The semiMarkov process and regeneration point technique are used to derive the expressions for some important reliability measures such as transition probabilities, mean sojourn times, mean time to system failure (MTSF), availability, busy period of the server for inspection, maintenance and repair, expected number of visits of the server and profit function. The graphical behavior of MTSF, availability and profit function have been observed for particular values of different parameters and costs.

\section{Notation}

$\mathrm{E}$

$\mathrm{O} / \mathrm{Cs}$

$\lambda$

$\mathrm{a} / \mathrm{b}$

$a_{1} / b_{1}$

$\mathrm{h}_{1}(\mathrm{t}) / \mathrm{H}_{1}(\mathrm{t})$

$\mathrm{g}(\mathrm{t}) / \mathrm{G}(\mathrm{t})$

$\mathrm{SU}_{\mathrm{m}}$

FUr /FUR

FUi /FUI
Set of regenerative states. The unit is in operative / cold standby mode.

Constant failure rate of the unit.

Probability that standby unit is operable/nonoperable.

Probability that the failed unit is repairable/ replaceable.

$\mathrm{pdf} / \mathrm{cdf}$ of inspection time of the unit.

pdf/cdf of repair time of the unit.

Standby unit under maintenance.

The unit is failed and under repair / under repair continuously from previous state. under inspection / under inspection continuously
The unit is failed and 


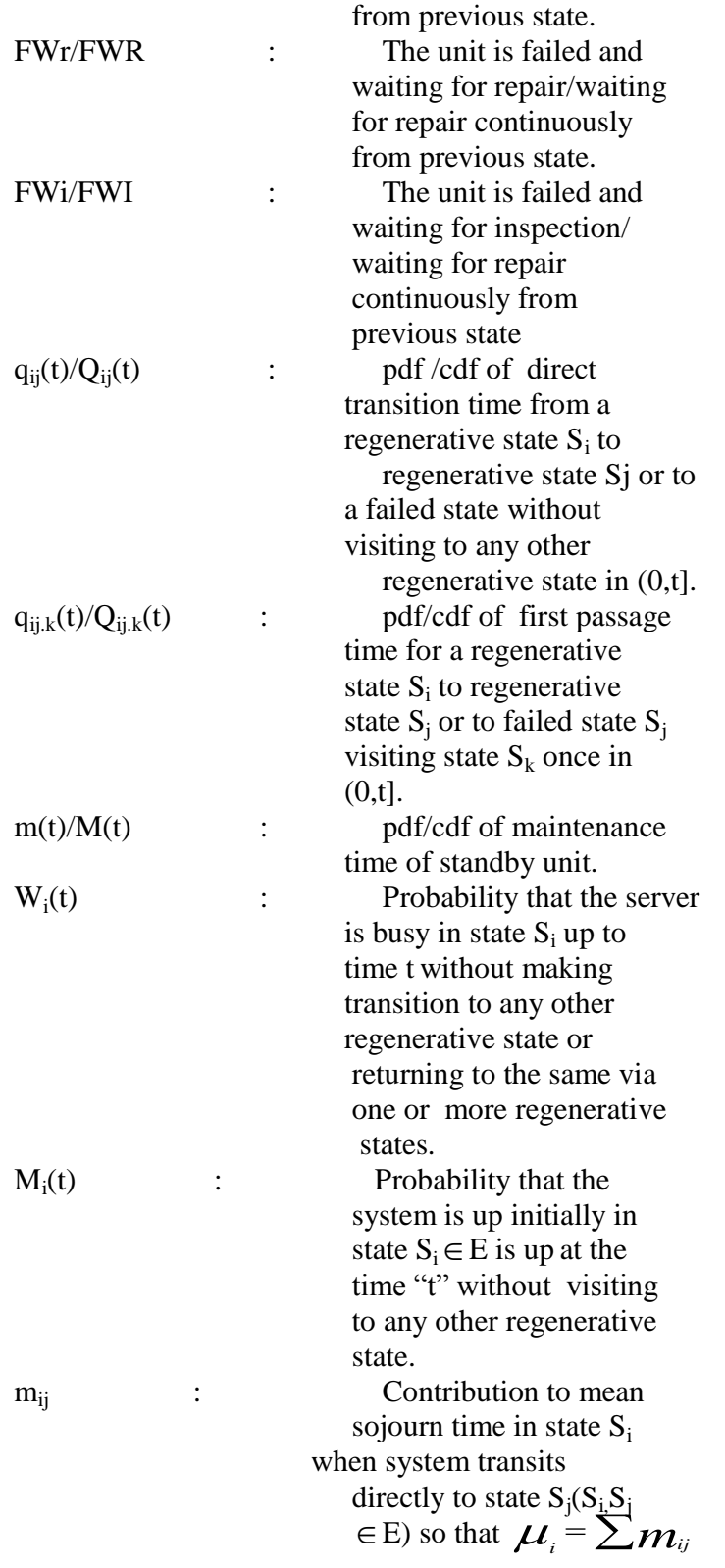

where

$$
m_{i j}=\int t d Q_{i j}(t)=-q_{i j}^{*^{\prime}}(0)
$$

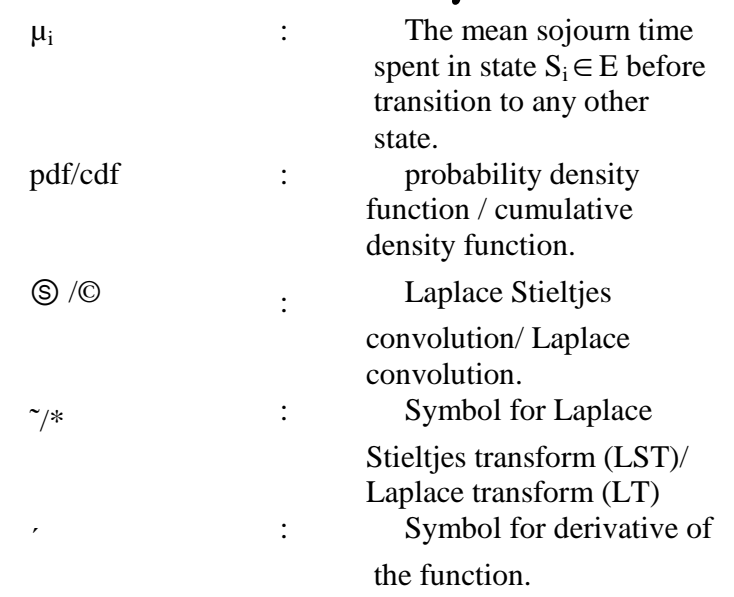

The possible transition states of the system model is shown in fig.1
The transition states $S_{0}, S_{1}, S_{2}$ and $S_{3}$ are regenerative and $S_{4}$, $\mathrm{S}_{5}$ and $\mathrm{S}_{6}$ are non- regenerative.

\section{TRANSITION PROBABILITIES AND MEAN SOJOURN TIMES}

Simple probabilistic considerations yield the following expressions the non-zero elements $\mathrm{p}_{\mathrm{ij}}$ are given by

$$
\begin{aligned}
& \mathrm{p}_{01}=\mathrm{a}, \quad \mathrm{p}_{02}=\mathrm{b}, \quad \mathrm{p}_{10}=b_{1} h_{1} *(\lambda), \\
& \mathrm{p}_{14}=1-h_{1} *(\lambda), \quad \mathrm{p}_{13}=a_{1} h_{1} *(\lambda), \\
& \mathrm{p}_{36}=1-g *(\lambda) \mathrm{p}_{41}=b_{1} h_{1} *(0), \\
& \mathrm{p}_{51}=\mathrm{p}_{61}=g *(0) .
\end{aligned}
$$

It can be verified that

$\mathrm{p}_{01}+\mathrm{p}_{02}=1 ; \mathrm{p}_{10}+\mathrm{p}_{13}+\mathrm{p}_{14}=1 ; \mathrm{p}_{10}+\mathrm{p}_{11.3}+\mathrm{p}_{14}=1 ; \mathrm{p}_{30}+\mathrm{p}_{36}=1, \mathrm{p}_{41}$ $+\mathrm{p}_{45}=1, \mathrm{p}_{21}=\mathrm{p}_{51}=\mathrm{p}_{61}=1$

The Mean Sojourn Times $\left(\mu_{\mathrm{i}}\right)$ in the State $S_{\mathrm{i}}$ are

$$
\begin{aligned}
& \mu_{0}=\mathrm{m}_{01}+\mathrm{m}_{02}=\frac{1}{\lambda}, \mu_{1}=\mathrm{m}_{10}+\mathrm{m}_{13}+\mathrm{m}_{14=} \frac{1-h^{*}(\lambda)}{\lambda} \\
& \mu_{2}=\mathrm{m}_{21}=-\mathrm{m}^{*}(0), \mu_{3}=\mathrm{m}_{30}+\mathrm{m}_{36=} \frac{1-g *(\lambda)}{\lambda} \\
& \mu_{4}=\mathrm{m}_{41}+\mathrm{m}_{45=}-h_{1} *^{\prime}(0), \\
& \mu_{5}=\mathrm{m}_{51=}-g^{* \prime}(0)=\mu_{6}=\mathrm{m}_{61}, \\
& \mu_{3}{ }^{\prime}=\mathrm{m}_{30}+\mathrm{m}_{31.6}=\frac{1}{\lambda}[1-g *(\lambda)] \cdot\left[1-\lambda g *^{\prime}(0)\right] \\
& \mu_{1}{ }^{\prime}=\mathrm{m}_{10}+\mathrm{m}_{13}+\mathrm{m}_{11.4}+\mathrm{m}_{11.45}= \\
& -h_{10} *(\lambda)-\left(1-h_{1} *(\lambda) h_{1} *^{\prime}(0)\left[a_{1} g^{*} 0\right)\right. \\
& \left.+a_{1} h^{*}(0) g^{* \prime}(0)+b_{1}\right]-h_{1} *^{\prime}(\lambda)\left[a_{1} h_{1} *^{\prime}(0) g *(0)+b_{1} h_{1} *(0)\right. \\
& +\frac{b_{1}}{\lambda}\left(1-h_{1} *(\lambda) \cdot h_{1} *(0)\right.
\end{aligned}
$$

\section{MEAN TIME TO SYSTEM FAILURE (MTSF)}

Let $\phi_{i}(t)$ be the cdf of first passage time from regenerative state $S_{i}$ to a failed state. Regarding the failed state as absorbing state, we obtain the following recursive relations for $\phi_{i}(t)$ :

$$
\begin{aligned}
& \phi_{0}(t)=Q_{01}(t) \text { (s) } \phi_{1}(t)+Q_{02}(t) \\
& \phi_{1}(t)=Q_{10}(t) \text { (s) } \phi_{0}(t)+Q_{13}(t) \text { (s) } \phi_{3}(t)+Q_{14}(t) \\
& \phi_{3}(t)=Q_{30}(t) \text { (s) } \phi_{0}(t)+Q_{36}(t)
\end{aligned}
$$

Taking LST of relations (1) and solving for $\tilde{\phi}_{0}(s)$, we have

$$
\tilde{\phi}_{0}(s)=\frac{\tilde{Q}_{02}(s)+\tilde{Q}_{01}(s) \tilde{Q}_{13}(s)}{1-\tilde{Q}_{01}(s) \tilde{Q}_{10}(s)}
$$

The mean time to system failure (MTSF) is given by

$$
M T S F=l_{s \rightarrow 0} \frac{1-\tilde{\phi}_{0}(s)}{s}=\frac{N}{D}=\frac{\mu_{0}+p_{01} \mu_{1}+p_{01} p_{13} \mu_{3}}{1-p_{01} p_{10}-p_{30} p_{13} p_{01}}
$$




\section{STEADY STATE AVAILABILITY}

Let $A_{i}(t)$ be the probability that the system is in up-state at instant ' $t$ ' given that the system entered regenerative state $S_{i}$ at $\mathrm{t}=0$. The recursive relations for $A_{i}(t)$ are given as:

$A_{0}(t)=M_{0}(t)+q_{01}(t) \subset \mathrm{A}_{1}(t)+q_{02}(t) \subset \mathrm{A}_{2}(t)$

$A_{1}(t)=M_{1}(t)+q_{10}(t) \subset \mathrm{A}_{0}(t)+q_{13}(t) \subset \mathrm{A}_{3}(t)$

$+\left(q_{11.4}(t)+q_{11.45}(t)\right) \subset A_{1}(t)$

$A_{2}(t)=q_{21}(t) \subseteq \mathrm{A}_{1}(t)$

$A_{3}(t)=M_{3}(t)+q_{30}(t) \subseteq \mathrm{A}_{0}(t)+q_{31.6}(t) \subseteq \mathrm{A}_{1}(t)$

Where $\quad M_{0}(t)=e^{-\lambda t}, \quad M_{1}(t)=e^{-\lambda t} \overline{H_{1}(t)}$

$M_{3}(t)=e^{-\lambda t} \overline{G(t)}$

Taking Laplace transform of the relations (4) and solving for $A_{0}{ }^{*}(s)$, we have

$A_{0}^{*}(\infty)=\operatorname{lt}_{s \rightarrow 0} A_{0}^{*}(s)=N_{1} / D_{1}$

$N_{1}=\left(p_{10}+p_{13} p_{30}\right) \mu_{0}+\mu_{1}+p_{13} \mu_{3}$,

$D_{1}=p_{13} \mu_{3}^{\prime}+\mu_{1}^{\prime}+\left(p_{02} \mu_{2}+\mu_{0}\right)\left(p_{10}+p_{13} p_{30}\right)$

\section{BUSY PERIOD OF THE SERVER DUE TO INSPECTION AND MAINTENANCE}

Let $B_{i}^{i m}(t)$ be the probability that the server is busy at instant' $t$ ' due to inspection and maintenance given that the system entered regenerative state $S_{i}$ at $t=0$. The recursive relations for $B_{i}^{i m}(t)$ are:

$$
\begin{aligned}
& B_{0}^{i m}(t)=q_{01}(t) @ B_{1}^{i m}(t)+q_{02}(t) @ \mathrm{~B}_{2}^{\mathrm{im}}(t) \\
& B_{1}^{i m}(t)=W_{1}^{i m}(t)+q_{10}(t) @ B_{0}^{i m}(t)+q_{13}(t) @ B_{3}^{i m}(t)+\left[q_{11.4}(t)\right. \\
& \left.+q_{11.45}(t)\right] \bigodot B_{1}^{i m}(t) \\
& \left.B_{2}^{i m}(t)=q_{20}(t)\right) \subset \mathrm{B}_{1}^{\mathrm{im}}(t) \\
& B_{3}^{i m}(t)=q_{30}(t) \Subset B_{0}^{i m}(t)+q_{31.6}(t) \Subset \mathrm{B}_{1}^{\mathrm{im}}(t)
\end{aligned}
$$

where

$$
W_{1}^{i m}(t)=e^{-\lambda t} \overline{H(t)}+\left[\lambda e^{-\lambda t} \mathrm{C} 1\right] \overline{H(t)}
$$

Taking LT of relations (5) and solving for $B_{0}^{i m^{*}}(s)$. The time for which server is busy due to inspection and maintenance in the long run is given by:

$B_{0}^{i m}(\infty)=\operatorname{lt}_{s \rightarrow 0} B_{0}^{i m^{*}}(s)=N_{2} / D_{1}$

where, $N_{2}=W_{1}^{i m^{*}}(0)$ and $\mathrm{D}_{1}$ is already defined.

\section{BUSY PERIOD OF THE SERVER DUE TO REPAIR}

Let $B_{i}^{r}(t)$ be the probability that the server is busy at instant' $t$ ' due to repair given that the system entered regenerative state $S_{i}$ at $t=0$. The recursive relations for $B_{i}^{r}(t)$ are as follows:

$$
\begin{aligned}
& B_{0}^{r}(t)=q_{01}(t) \Subset \mathrm{B}_{1}^{\mathrm{r}}(t)+q_{02}(t) \Subset \mathrm{B}_{2}^{\mathrm{r}}(t) \\
& B_{1}^{r}(t)=q_{10}(t) @ B_{0}^{r}(t)+q_{13} @ B_{3}^{r}(t) \\
& +\left[q_{11.4}(t)+q_{11.45}(\mathrm{t})\right] \Subset B_{1}^{r}(t) \\
& B_{2}^{r}(t)=q_{21}(t) \subset \mathrm{B}_{1}^{\mathrm{r}}(t) \\
& B_{3}^{r}(t)=W_{3}^{r}(t)+q_{30}(t)\left(B_{0}^{r}(t)+q_{31.6} \subset \mathrm{B}_{1}^{\mathrm{r}}(t)\right.
\end{aligned}
$$

where$$
W_{3}^{r}(t)=e^{-\lambda t} \overline{G(t)}+\left[\lambda e^{-\lambda t} \mathrm{C} 1\right] \overline{G(t)}
$$

Taking LT of relations (6) and solving for $B_{0}^{r^{*}}(s)$.

The time for which server is busy due to repair in the long run is given by

$$
\begin{aligned}
& B_{0}^{r}(\infty)=\operatorname{lt}_{s \rightarrow 0} B_{0}^{r^{*}}(s)=N_{3} / D_{1} \\
& \text { where } N_{3}=p_{13} W_{3}^{r^{*}}(0) \text { and } \mathrm{D}_{1} \text { is already defined. }
\end{aligned}
$$

\section{EXPECTED NUMBER OF VISITS BY THE SERVER}

Let $N_{i}(t)$ be the expected number of visits by the server at instant ' $t$ ' given that the system entered regenerative state $S_{i}$ at $\mathrm{t}=0$. The recursive relations for $N_{i}(t)$ are:

$$
\begin{aligned}
& N_{0}(t)=Q_{01}(t) \text { (s) }\left[1+N_{1}(t)\right]+Q_{02}(t) \text { (s) } N_{2}(t) \\
& N_{1}(t)=Q_{10}(t) \text { (s) } N_{0}(t)+Q_{13}(t) \text { (s) } \\
& N_{3}(t)+\left[Q_{11.4}(t)+Q_{11.45}(t)\right] \text { (s) } N_{1}(t) \\
& N_{2}(t)=Q_{21}(t) \text { (s) } N_{1}(t) \\
& N_{3}(t)=Q_{30}(t) \text { (s } N_{0}(t)+Q_{31.6}(t) \text { (s) } N_{1}(t)
\end{aligned}
$$

Taking LST of relations (7) and Solving for $N_{0}^{*}(s)$. The expected number of visits by the server is given by

$$
\begin{aligned}
& N_{0}(\infty)=\operatorname{lt}_{s \rightarrow 0} N_{0}^{*}(s)=N_{4} / D_{1} \\
& N_{4}=p_{01} \cdot\left[p_{10}+p_{13} p_{30}\right] \text { and } \mathrm{D}_{1} \text { is already defined. }
\end{aligned}
$$

\section{PROFIT ANALYSIS}

The profit incurred to the system in steady state is given by $P=K_{0} A_{0}-K_{1} B_{0}^{i m}-K_{2} B_{0}^{i m}-K_{3} N_{0}$

Where

$K_{0}=$ Revenue per unit up-time of the system.

$K_{1}=$ Cost per unit time for which server is busy due to inspection / maintenance.

$K_{2}=$ Cost per unit time for which server is busy due to repair.

$K_{3}=$ Cost per unit time for which server is visited.

\section{PARTICULAR CASE}




$$
\text { If we have } g(t)=\alpha e^{-\alpha t} \quad, \quad h_{1}(t)=\beta_{1} e^{-\beta_{1} t}
$$

$m(t)=\gamma e^{-x}$

We obtain the following results:

$N=\frac{(\alpha+\lambda) \lambda a+(\alpha+\lambda)(\beta+\lambda)+a a_{1} \beta \lambda}{\lambda(\alpha+\lambda)(\beta+\lambda)}$

$D=\frac{(\alpha+\lambda)(\beta+\lambda)-a b_{1} \beta(\alpha+\lambda)-a a_{1} \alpha \beta}{(\alpha+\lambda)(\beta+\lambda)}$

$N_{1}=\frac{1}{\alpha+\lambda} \cdot \frac{a_{1} \cdot \beta}{\beta+\lambda}+\frac{1}{\beta+\lambda}+\frac{1}{\lambda}\left(1+\frac{b_{1} \cdot \beta}{\beta+\lambda}+\frac{a_{1} \cdot \beta}{\beta+\lambda} \cdot \frac{\alpha}{\alpha+\lambda}\right)$,

$N_{2}=\frac{1}{\beta}, N_{3}=\frac{1}{\alpha} \cdot \frac{a_{1} \cdot \beta}{\beta+\lambda}$,

$N_{4}=a\left[\frac{b_{1} \cdot \beta}{\beta+\lambda}+\frac{a_{1} \cdot \beta}{\beta+\lambda} \cdot \frac{\alpha}{\alpha+\lambda}\right]$

$D_{1}=\frac{\alpha}{\alpha+\lambda}\left[\frac{1}{\lambda}+\frac{1}{\beta}+\frac{b}{\gamma}\right]+\frac{1}{\alpha}$

\section{CONCLUSION}

The graphical behavior of some performance measures of a stochastic model has been observed for different values of parameters as shown in figures 2, 3 and 4 . It is seen that mean time to system failure (MTSF), availability and profit function go on decreasing with the increase of failure rate $(\lambda)$ while they increase with the increase of repair rate $(\alpha)$, inspection rate $\left(\beta_{1}\right)$ and maintenance rate $(\gamma)$. Furthermore, performance measures decline if standby unit has more chances of its maintenance before operation. Again, system becomes more profitable by making replacement of the failed unit rather than its repair. Thus, on the basis of the results obtained for a particular case it is concluded that a system in which cold standby unit has more chances of its maintenance before operation can be made more profitable by making replacement of the failed unit by new one instead of its repair.

\section{REFERENCES}

[1] Gupta, Rakesh and Mumtaz, S.Z., 1996. Stochastic analysis of a two-unit cold stand by system with maximum repair time \& correlated failures and repairs. Journal of Quality in Maintenance Engineering, Vol.2 (3), pp.66-76.

[2] Yadavalli, V.S.S., Chanderasekhar and Natarajan, R., 2004. A study on two-unit standby system with Erlangian repair time. Asia-Pacific Journal of Operational Research, Vol. 21(3), pp. 271-277.

[3] Bao, X., and Cui, L., 2012. A study on reliability for a two-item cold standby Markov repairable system with neglected failures. Communications in Statistics-Theory and Methods, Vol. 41(21), pp. 3988-3999.

[4] Kumar, J., Kadyan, M. S., and Malik, S. C., 2012. Cost analysis of a two unit cold standby system subject to degradation, inspection and priority. Eksploatacja i Niezawodnosc - Maintenance and Reliability, Vol. 14(4), pp. 278-283.

[5] Malik, S.C. and Barak, Sudesh K., 2013. Reliability Measures of a Cold Standby System with Preventive Maintenance and Repair. International Journal of Reliability, Quality and Safety Engineering, Vol. 20(6), DOI: $10.1142 / \mathrm{S} 0218539313500228$.

[6] Malik, S.C., 2013. Reliability Modeling of a Computer System with Preventive Maintenance and Priority Subject to Maximum Operation and Repair Times. International Journal of System Assurance Engineering and Management, Vol. 4(1), pp.94-100.

[7] Yusuf Ibrahim and Yusuf Bashir, 2014. Analysis of some reliability characteristic between two dissimilar redundant system with replacement at common cause failure. International Journal of Advance Statistic \& Probability, Vol. 2 (1), pp. 1-14.

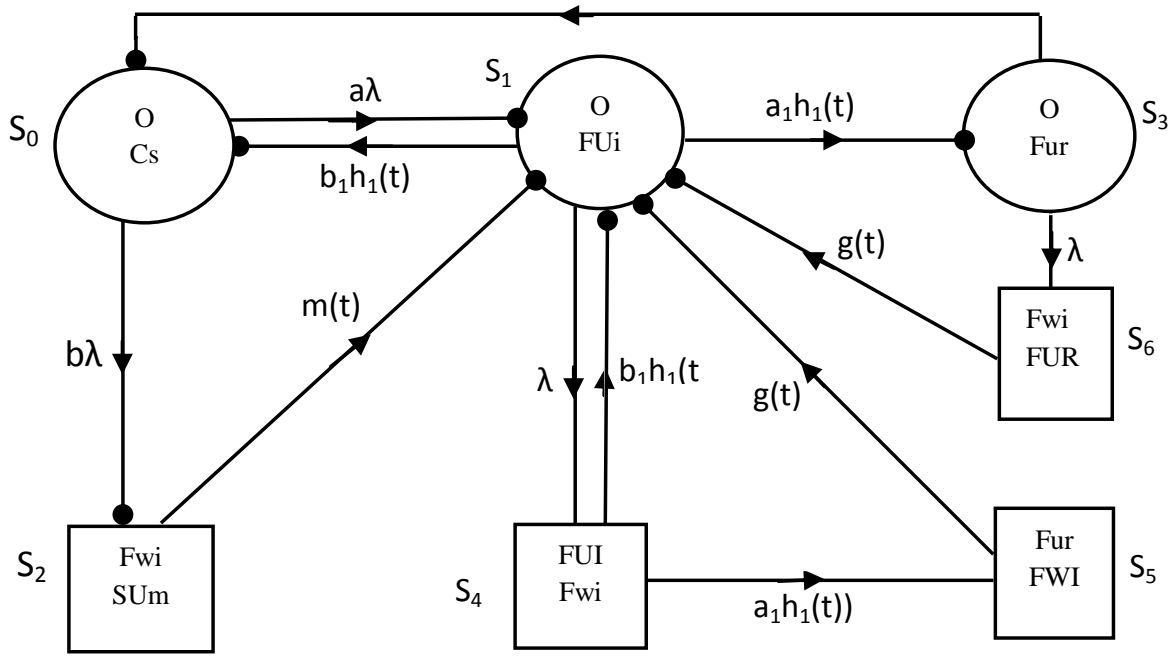

Regenerative Point

up state

failed state

Fig.1 


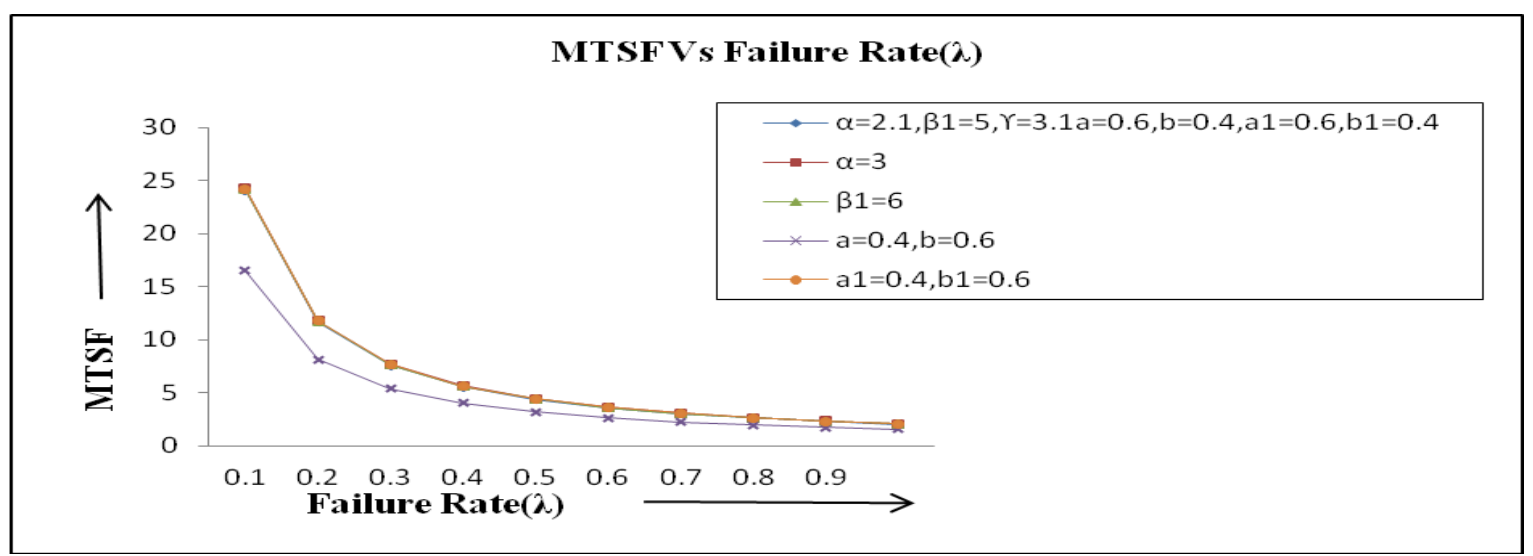

Fig. 2

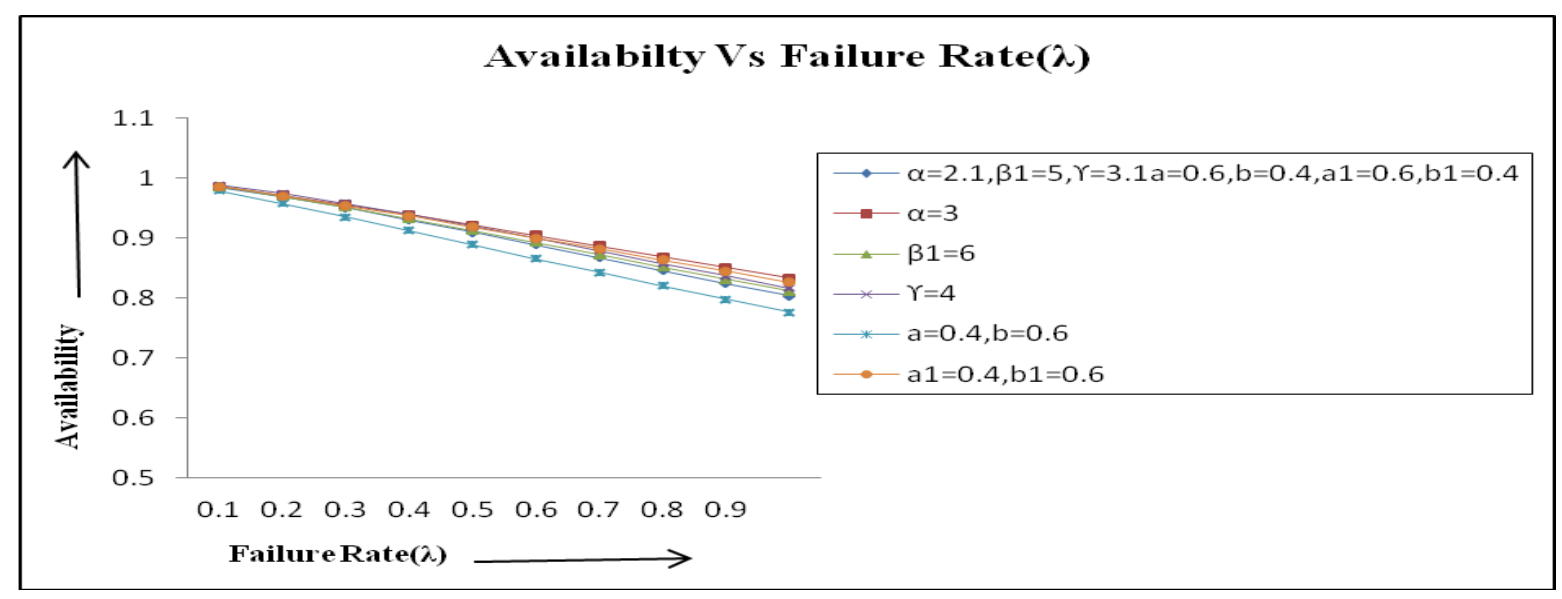

Fig. 3

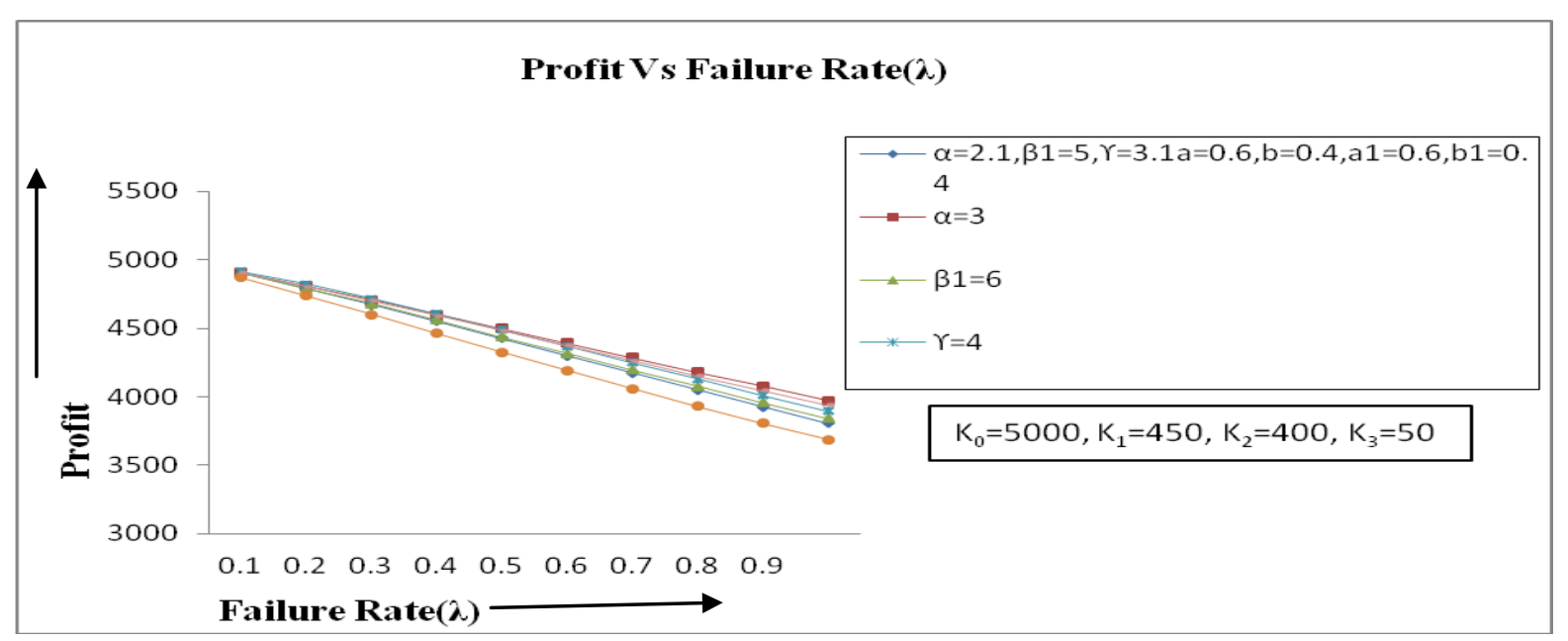

Fig. 4 